\title{
Human Fecal Metabolome Reflects Differences in Body Mass Index, Physical Fitness, and Blood Lipoproteins in Healthy Older Adults
}

\author{
Mengni Cui ${ }^{1}{ }^{D}$, Alessia Trimigno ${ }^{1}\left(\mathbb{D}\right.$, Josue L. Castro-Mejía ${ }^{2}$, Søren Reitelseder ${ }^{3}$, Jacob Bülow ${ }^{3}$, \\ Rasmus Leidesdorff Bechshøft ${ }^{3}$, Dennis Sandris Nielsen ${ }^{2} \mathbb{D}$, Lars Holm ${ }^{3,4} \mathbb{D}$, Søren Balling Engelsen ${ }^{1, *} \mathbb{D}$ \\ and Bekzod Khakimov ${ }^{1, *(1)}$
}

1 Chemometrics and Analytical Technology Section, Department of Food Science University of Copenhagen Rolighedsvej 26, 1958 Frederiksberg C, Denmark; mengni@food.ku.dk (M.C.); alessia@food.ku.dk (A.T.)

2 Food Microbiology \& Fermentation Section, Department of Food Science University of Copenhagen Rolighedsvej 26, 1958 Frederiksberg C, Denmark; jcame@food.ku.dk (J.L.C.-M.); dn@food.ku.dk (D.S.N.)

3 Institute of Sports Medicine, Department of Orthopedic Surgery, Bispebjerg and Frederiksberg Hospital, Nielsine Nielsens Vej 11, 2400 Copenhagen, Denmark; s.reitelseder@gmail.com (S.R.); jacob.buelow.02@regionh.dk (J.B.); r.bechshoeft@gmail.com (R.L.B.); L.Holm@bham.ac.uk (L.H.)

4 School of Sport, Exercise and Rehabilitation Sciences, University of Birmingham, Edgbaston, Birmingham B15 2TT, UK

check for updates

Citation: Cui, M.; Trimigno, A.; Castro-Mejía, J.L.; Reitelseder, S.; Bülow, J.; Bechshøft, R.L.; Nielsen, D.S.; Holm, L.; Engelsen, S.B.; Khakimov, B. Human Fecal Metabolome Reflects Differences in Body Mass Index, Physical Fitness, and Blood Lipoproteins in Healthy Older Adults. Metabolites 2021, 11, 717. https://doi.org/10.3390/ metabo11110717

Academic Editors: Allison B. Reiss and Joshua De Leon

Received: 17 September 2021

Accepted: 15 October 2021

Published: 21 October 2021

Publisher's Note: MDPI stays neutral with regard to jurisdictional claims in published maps and institutional affiliations.

Copyright: (c) 2021 by the authors. Licensee MDPI, Basel, Switzerland. This article is an open access article distributed under the terms and conditions of the Creative Commons Attribution (CC BY) license (https:/ / creativecommons.org/licenses/by/ $4.0 /)$.
* Correspondence: se@food.ku.dk (S.B.E.); bzo@food.ku.dk (B.K.); Tel.: +45-3533-3205 (S.B.E.); +45-3532-8184 (B.K.)

\begin{abstract}
This study investigated how body mass index (BMI), physical fitness, and blood plasma lipoprotein levels are related to the fecal metabolome in older adults. The fecal metabolome data were acquired using proton nuclear magnetic resonance spectroscopy and gas chromatography-mass spectrometry on 163 healthy older adults (65-80 years old, 80 females and 83 males). Overweight and obese subjects (BMI $\geq 27$ ) showed higher levels of fecal amino acids (AAs) (valine, alanine, and phenylalanine) compared to normal-weight subjects $(\mathrm{BMI} \leq 23.5)$. Adults classified in the high-fitness group displayed slightly lower concentrations of fecal short-chain fatty acids, propionic acid, and AAs (methionine, leucine, glutamic acid, and threonine) compared to the low-fitness group. Subjects with lower levels of cholesterol in low-density lipoprotein particles (LDLchol, $\leq 2.6 \mathrm{mmol} / \mathrm{L}$ ) displayed higher fecal levels of valine, glutamic acid, phenylalanine, and lactic acid, while subjects with a higher level of cholesterol in high-density lipoprotein particles (HDLchol, $\geq 2.1 \mathrm{mmol} / \mathrm{L}$ ) showed lower fecal concentration of isovaleric acid. The results from this study suggest that the human fecal metabolome, which primarily represents undigested food waste and metabolites produced by the gut microbiome, carries important information about human health and should be closely integrated to other omics data for a better understanding of the role of the gut microbiome and diet on human health and metabolism.
\end{abstract}

Keywords: human fecal metabolome; BMI; fitness; lipoproteins; ${ }^{1} \mathrm{H}$ NMR; GC-MS; SCFA; Signature Mapping

\section{Introduction}

Human fecal matter is a waste product of food digestion and mainly contains water, undigested food remnants, including nitrogen and protein matter, carbohydrates, lipids, and hundreds of small molecular metabolites, as well as bacteria [1]. Fecal matter is also a rich source of metabolic information and the noninvasive sampling has increased interest toward exploring the human fecal metabolome as a function of diet, diseases, and other lifestyle conditions [1,2]. Recent studies have shown close relationships between the dynamics of the fecal metabolome and gut microbiome functions [3,4]. Numerous studies have related changes in the fecal metabolome with chronic and genetic diseases and linked 
them with the gut microbiome functions [5]. The impacts of various chronic diseases on the human fecal metabolome, including bowel disease [6], obesity [7], type-2 diabetes (T2D) [8], liver diseases [9,10], and cardiovascular diseases (CVDs) [11], have shown the potentiality of fecal metabolomics for better understanding of the pathologies of these diseases. There also evidence suggesting that the majority of the above-mentioned chronic diseases are largely influenced by BMI [12], lifestyles (e.g., diet, exercise) [13], physical fitness [14], and clinical blood parameters such as plasma concentrations of lipoproteins (LPs) [15].

This paper reports a fecal metabolomics study performed on healthy older adults using proton $\left({ }^{1} \mathrm{H}\right)$ nuclear magnetic resonance (NMR) spectroscopy [16] and untargeted gas chromatography-mass spectrometry (GC-MS) [17]. In addition, lipoprotein profiling of plasma samples was performed using ${ }^{1} \mathrm{H}$ NMR spectroscopy $[18,19]$. The study showed weak but significant systematic variations in the human fecal metabolome related to BMI, physical fitness, and blood plasma LP levels. The subjects of this study are healthy older adults, include 83 males $(70+/-5$ age) and 80 females $(70+/-5$ age), recruited in the Counteracting Age-related Loss of Skeletal Muscle Mass (CALM) study [20]. The CALM study was designed to investigate the effect of increased protein supplementation and physical exercise on the general health and well-being of community-dwelling relatively healthy older Danes. The present paper scrutinized the fecal metabolome data obtained on all subjects at the baseline, pre-intervention time. Apart from physical fitness, the study showed fecal metabolic fluctuations according to the health-indicating parameters including BMI and plasma LP levels. The distributions of these parameters, including upper and lower limits of specific clinical parameters determined by the Danish Health Care system [21], are illustrated in Figure 1. Physical fitness in the CALM study was determined as a function of three variables, including (1) chair-rise test, (2) body composition measured with dexa-scan, and (3) BMI [14]. Using this strategy, all 163 CALM subjects at baseline were divided into two groups: low fitness (LF) and high fitness (HF).
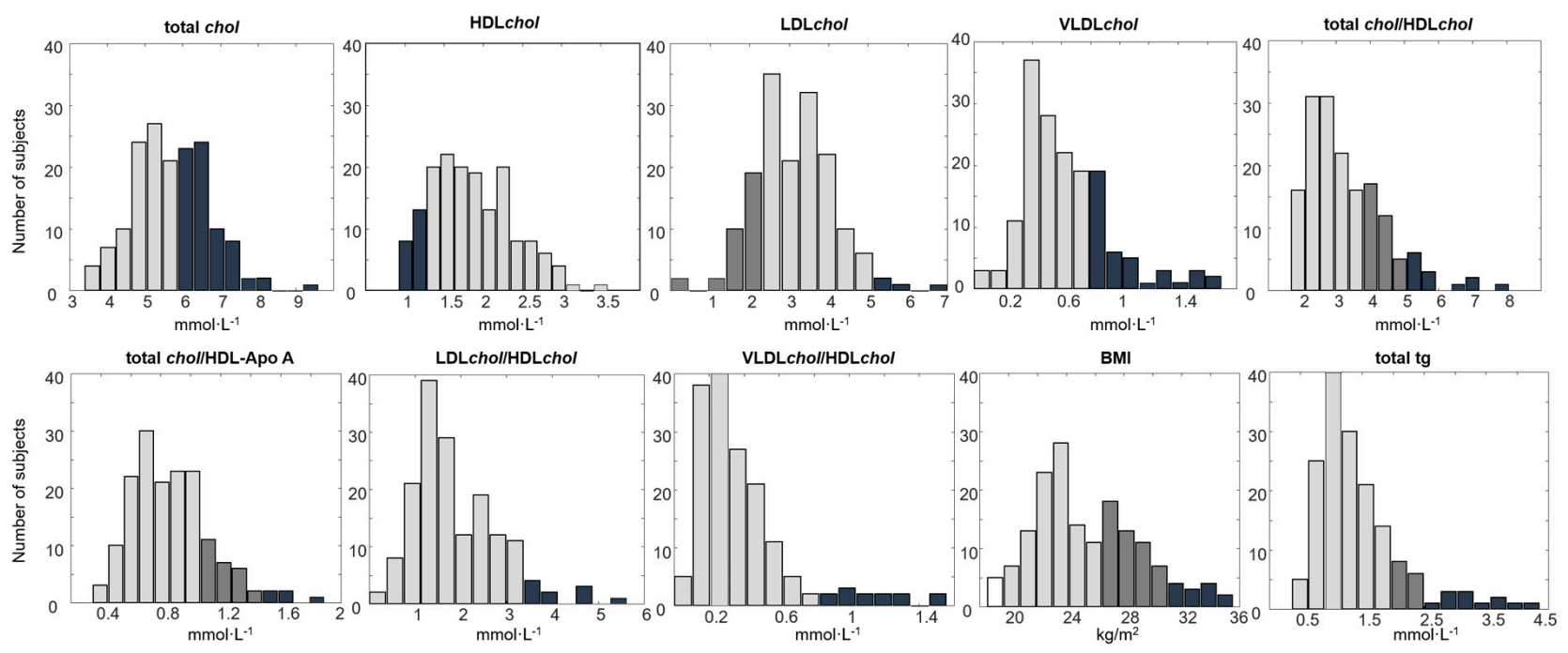

Figure 1. Histograms showing the distributions of human blood lipoproteins (LPs), their ratios, and BMI across the 163 subjects included in this study. The four colors in the figure represent four levels of risk thresholds set in the Danish healthcare system [21], including below the normal range (white), normal range (light grey), borderline range (dark grey), and under risk (black). The unit for LP levels and total tg is $\mathrm{mmol} / \mathrm{L}$, and that for $\mathrm{BMI}$ is $\mathrm{kg} / \mathrm{m}^{2}$. $\mathrm{BMI}=$ body mass index, HDLchol = high-density lipoprotein cholesterol, VLDLchol = very-low-density lipoprotein cholesterol, LDLchol = low-density lipoprotein cholesterol, total tg= total triglycerides, total chol = total cholesterol and total chol/HDLchol, HDL-Apo A = high-density lipoprotein-Apolipoprotein A.

Previous studies have reported that both BMI and physical fitness are closely related to the levels of blood LP [22]. Rashid et al. reported that physical exercise is an effective way for raising the HDLchol level [23]. Van Gaal et al. showed that BMI in pre- 
menopausal women is negatively correlated to the plasma levels of HDLchol and positively correlated to LDLchol [24]. Cuesta-Zuluaga et al. found a close relationship between gut microbiome dysbiosis, obesity, CVD risk factors, and fecal short-chain fatty acids (SCFAs) levels in community-dwelling adults [25]. However, the relationships between the human fecal metabolome, BMI, physical fitness, and CVD risk factors such as LPs are not well characterized, and the present study aimed to improve this knowledge gap.

\section{Results}

\subsection{Human Fecal Metabolome of Older Adults}

The human fecal metabolome was investigated by employing two analytical platforms, namely ${ }^{1} \mathrm{H}$ NMR spectroscopy and GC-MS. Absolute concentrations of 36 human fecal metabolites and the relative concentrations of 76 unknown spin systems were quantified from ${ }^{1} \mathrm{H}$ NMR spectra, as described previously [16]. The mean absolute concentrations of the 36 human fecal metabolites are reported in Cui et al. [26]. These fecal metabolites covered five SCFAs, 13 AAs, five organic acids (OAs), two sugars, namely glucose and galactose, one nucleobase (uracil), and 11 other common metabolites, including ethanol, trimethylamine, methanol, carnitine, glycerol, guanidinoacetic acid, betaine, xanthine, acetone, and formic acid (Table S1, Cui et al., 2021). SCFAs and AAs were found to have the highest concentration in fecal metabolites in all subjects, regardless of sex, age, and fitness. Among the SFCAs, acetic acid was found to have the highest concentration, followed by propionic acid. Isoleucine was found to have the highest concentration among the AAs.

The GC-MS determined fecal metabolome included 149 peaks. Of these, 138 were identified at level 2 or 3 according to the Metabolomics Standards Initiative [27] using electron ionization-mass spectrometry (EI-MS) and retention index (RI)-based matching. A total of 49 metabolites were identified at level 2 (EI-MS match of $>80 \%$ and $\Delta$ RI of $<50$ ), whereas 89 metabolites were identified at level 3, to a metabolite class level or to a metabolite candidate level with limited confidence (EI-MS match of $>65 \%$ ). Fecal metabolites detected by GC-MS represented six molecular classes, including 14 AAs, 34 fatty acids (FAs), 19 sugars, 30 OAs, 11 sugar alcohols, and 47 other metabolites, including esters, uridine, and glucosamine (Table S1). In addition, metabolites such as derivatives of SCFAs, including 2,2-dimethyl-3-hydroxybutanoic acid and 2-oxo-propanoate, and OAs such as benzeneacetic acid, mercaptoacetic acid, and gluconic acid, and a few other level 3-identified metabolites in Table S1 have not been previously reported in human fecal matter and require further validation in new independent studies.

\subsection{Associations between the Human Fecal Metabolome and Physical Fitness}

In a previous study, physical fitness was defined from three variables, including the chair-rise test, body composition measured with dexa-scan and BMI [14]. In this study, the 163 older adults were divided into two groups according to physical fitness, 73 LF and $90 \mathrm{HF}$. In order to explore the effects of physical fitness, both univariate and multivariate analyses were carried out on the human fecal metabolome datasets generated using NMR and GC-MS. Multivariate data analysis methods, PCA and PLS-DA, revealed no clear differences in the fecal metabolomes of LF and HF groups. However, univariate analysis by means of one-way analysis of variance (ANOVA) revealed that 11 and 17 metabolites, quantified from NMR and GC-MS, respectively, were significantly different between LF and HF participants (Table S2). These metabolites included AAs such as leucine, isoleucine, valine, alanine, methionine, glutamic acid, sarcosine, threonine, phenylalanine, glycine, and other metabolites such as propionic acid, oxalic acid, nicotinic acid, lactic acid, cyclohexanecarboxylic acid, xylopyranose, and pentanol. Box plots of the six most significant metabolites, including leucine, methionine, glutamic acid, threonine, propionic acid, and lactic acid, are shown in Figure 2. All these metabolites showed a slight increase in their mean concentrations in the fecal samples of LF subjects. Propionic acid and methionine were the two most significant metabolites, displaying the greatest effect size ( $>4 \%)$. In addition, one-way ANOVA was applied to test the significance of the fitness effect in males 
and females separately (Table S3). The results indicated that methionine and threonine had significantly higher concentrations in the LF males compared to HF males, while fecal levels of propionic acid, malic acid, and fumaric acid were higher in LF females compared to HF females. A total of 13 metabolites detected using GC-MS, including ethenyl acetate, oxalic acid, 2-hydroxyisovaleric acid, and arabinopyranose, showed significantly increased concentrations in HF males compared to LF males. Seven metabolites, such as carbodiimide, oxalic acid, glutaric acid, and xylopyranose, were elevated in LF females compared to HF females. Ethenyl acetate $(p$-value $=0.006$, effect size $=10.6 \%)$ and oxalic acid ( $p$-value $=0.022$, effect size $=7.7 \%$ ) were the two metabolites with the largest effect size related to the fitness effect when studying males and females separately, and they were higher in HF males and females compared to the LF groups.
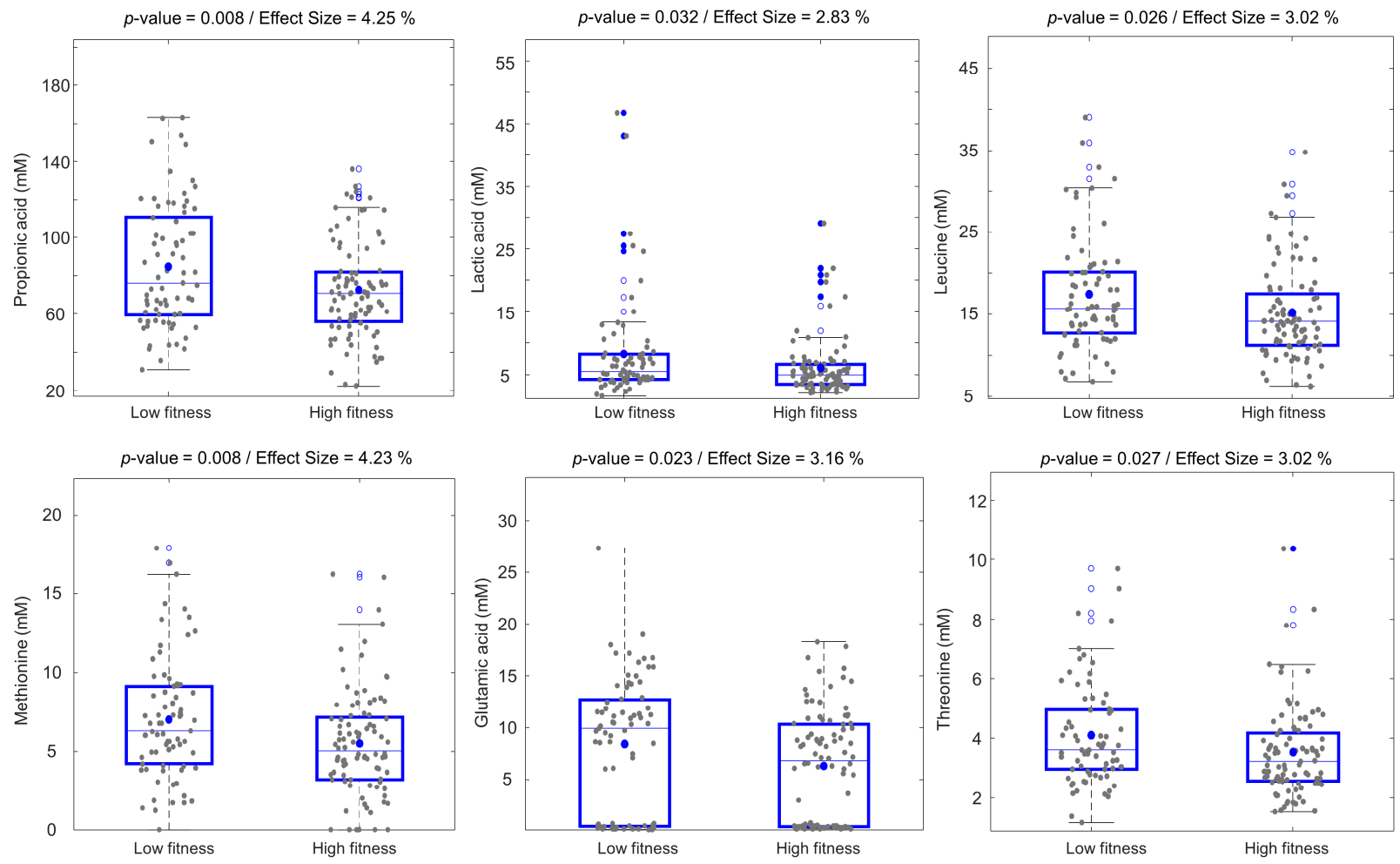

Figure 2. Boxplots of selected human fecal metabolites detected using ${ }^{1} \mathrm{H}$ NMR spectroscopy that are found to be different between low- and high-fitness subjects (low fitness, $n=73$; high fitness, $n=90)$ ). $p$-values and effect sizes (\%) are calculated from ANOVA. The horizontal lines and dots inside the boxes represent median and mean values, respectively. Blue circles and blue-filled circles correspond to quartiles $>75 \%$ and $95 \%$, respectively.

\subsection{Fecal Metabolic Differences between Overweight/Obese and Normal BMI Older Adults}

BMI is an important anthropometric factor reflecting human health and well-being [28]. Although BMI was one of the factors included for dividing older adults into the low- and high-physical-fitness groups, investigating the effect of BMI alone on the individual human fecal metabolic differences is of interest to understand the differences in the function of human gut microbiota in relatively overweight/obese and normal-BMI older adults. Therefore, all subjects were sorted according to BMI, and the bottom $1 / 3$ tertile (BMI $<23.5$, $n=54)$ and the top $1 / 3$ tertile $(\mathrm{BMI}>27, n=55)$ subjects were classified as low-BMI and high-BMI groups, respectively. As in the case of physical fitness, the human fecal metabolomics data were then evaluated with respect to BMI groups using univariate and multivariate data analysis methods. No systematic differences were found between the fecal metabolic profiles of low-BMI and high-BMI subjects using multivariate data analysis. In contrast, one-way ANOVA identified 14 metabolites from the ${ }^{1} \mathrm{H}$ NMR analysis that were significantly different between the two BMI groups (Table S4). These metabolites were AAs 
(leucine, valine, alanine, lysine, glycine, phenylalanine, methionine, tyrosine, glutamic acid, and isoleucine), SCFAs (propionic acid and pyruvic acid), uracil, and xanthine (Figure 3). All these metabolites displayed higher average concentrations in the high-BMI group. This trend remained constant when analyzing males and females separately (Table S5). In addition, 12 metabolites displayed significantly increased concentrations in high-BMI males compared to low-BMI males, and the majority of these were also found to be higher in highBMI subjects when evaluating both sexes together (Table S4). Three metabolites, glutamic acid, malic acid, and fumaric acid, were found to be higher in the high-BMI-females group compared to the low-BMI-females group.
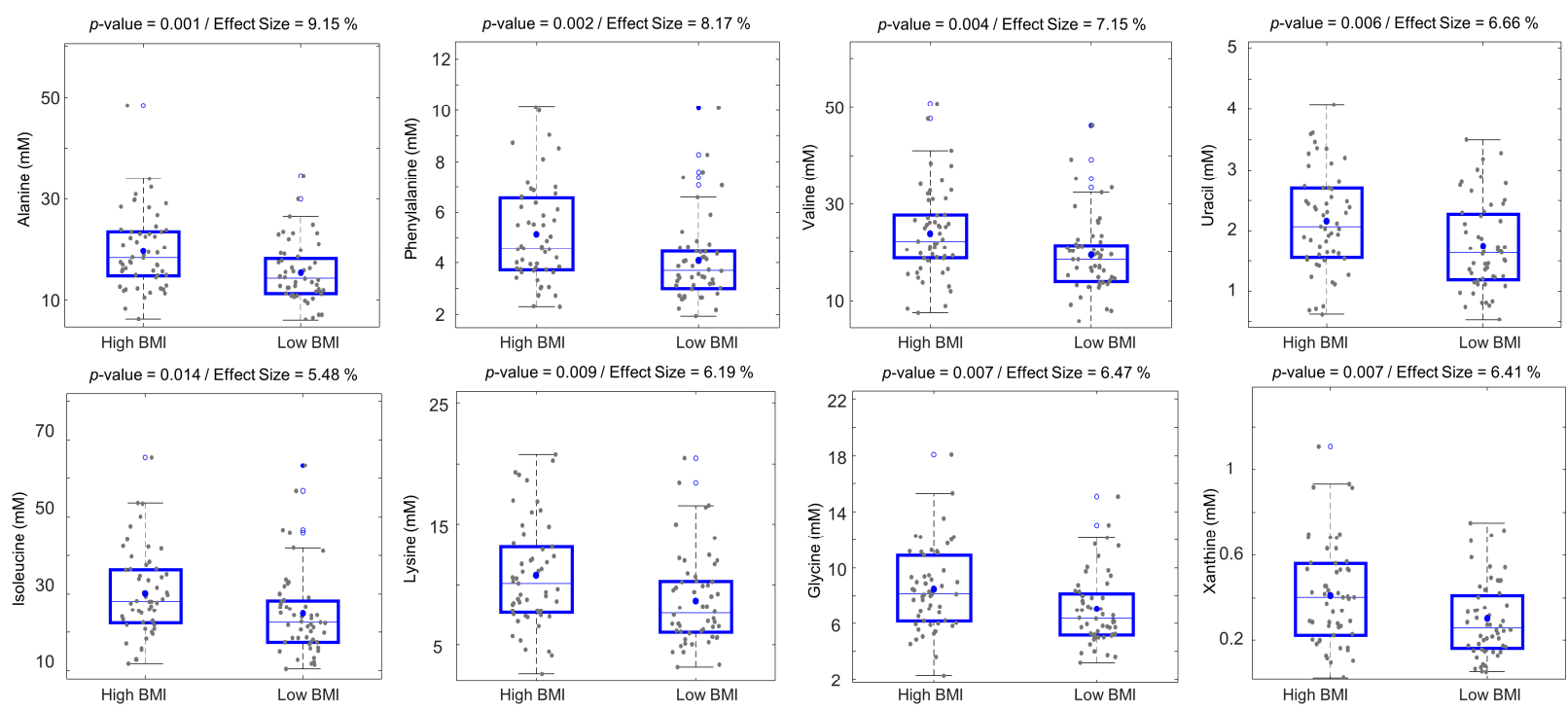

Figure 3. Boxplots of selected human fecal metabolites that are different between low-BMI $(<23.5, n=54)$ and high-BMI $(>27, n=55)$ groups. $p$-values and effect sizes $(\%)$ were calculated from ANOVA. The horizontal lines and dots inside the boxes represent median and mean values, respectively. Blue circles and blue-filled circles correspond to quartiles $>75 \%$ and $95 \%$, respectively.

\subsection{Associations between Blood Lipoproteins and the Fecal Metabolome}

The relationship between human blood plasma LP and the fecal metabolome is largely limited to studies investigating fecal cholesterol excretion [29], while untargeted fecal metabolomics has, to our knowledge, not been explored in the context of LP distribution in blood. Accordingly, human fecal metabolic differences in older adults were explored according to their plasma levels of cholesterol (chol), HDLchol, LDLchol, very-low-density lipoproteins particles (VLDLchol), and HDL-Apolipoprotein A (Apo A), and their ratios. First, using all subjects, Pearson's correlation coefficients were calculated between all human fecal metabolites and plasma LPs, including total chol, HDLchol, LDLchol, VLDLchol, and chol ratios, such as total chol/HDLchol, total chol/HDL-Apo A, LDLchol/HDLchol, and VLDLchol/HDLchol, to evaluate any possible correlation (Table S6). The results indicated that the level of total chol displayed a weak negative correlation with the fecal concentrations of lactic acid $(r$-value $=-0.2, p$-value $=0.006)$, ethanol $(r$-value $=-0.19$, $p$-value $=0.02)$, and formic acid $(r$-value $=-0.19, p$-value $=0.02)$. The ratio of total chol to HDL-Apo A also showed weak negative correlations with the fecal concentrations of isoleucine $(r$-value $=-0.17, p$-value $=0.03)$, methionine $(r$-value $=-0.17, p$-value $=0.03)$, threonine $(r$-value $=-0.18, p$-value $=0.024)$, uracil $(r$-value $=-0.18, p$-value $=0.02)$, and phenylalanine $(r$-value $=-0.16, p$-value $=0.04)$. The same correlation analysis was performed on sex or BMI-stratified subgroups. The distribution plots (Figure S1) represent an overview of the $r$-values calculated from the correlations between fecal metabolite levels of males, females, low-BMI, and high-BMI groups with the eight different selected fractions of cholesterols. The results showed that all $r$-values of the six groups were distributed 
in the range of -0.4 to 0.4 . The distribution plots of Figure 4 and heatmaps of Figure S2 represent the Pearson's correlation coefficients calculated between eight LP variables and 36 fecal metabolites in the six different subpopulations, including LF, HF, males, females, low BMI, and high BMI. The results indicated that correlations of fecal metabolites with plasma LPs were different when subjects were stratified according to fitness, sex, and BMI. Fecal metabolites of HF subjects showed more positive correlations with HDLchol level than in LF subjects, and similarly, HF subjects displayed more negative correlations with total chol. Fecal metabolites in males displayed more negative correlations with levels of total chol, LDLchol, and total chol/HDL-Apo A than in females. Low-BMI subjects showed greater positive correlations between their fecal levels of valeric acid and glucose with plasma total chol, as well as between the level of carnitine and HDLchol, when compared to high-BMI subjects, while high-BMI subjects showed significant negative correlations between fecal levels of threonine, betaine, and isoleucine and the total chol/HDL-Apo A ratio. Similarly, plasma levels of HDLchol were found to be at higher levels in HF subjects, whilst LF subjects displayed higher concentrations of LDLchol and IDLchol cholesterol fractions (Trimigno et al., in press).

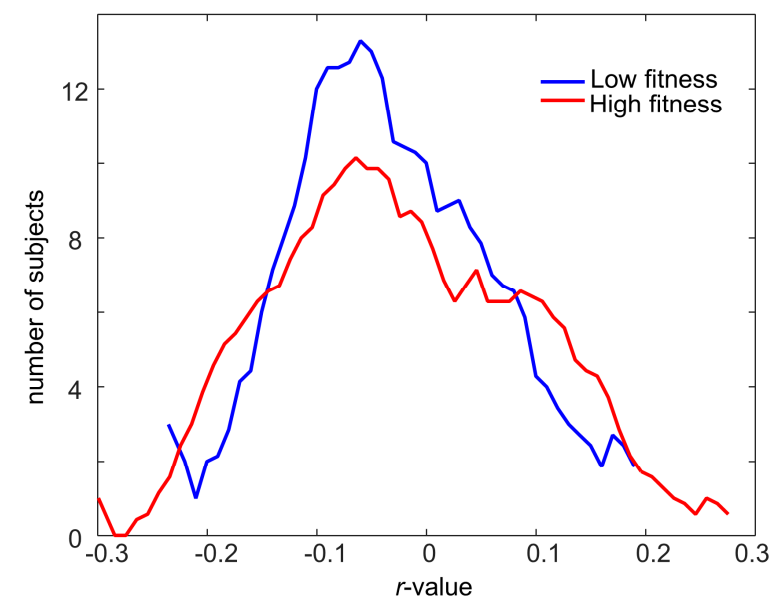

Figure 4. Distribution of Pearson's correlation coefficients ( $r$-values) calculated between 36 fecal metabolites including valeric acid, isovaleric acid, leucine, isoleucine, valine, propionic acid, ethanol, lactic acid, alanine, butyric acid, acetic acid, methionine, acetone, glutamic acid, pyruvic acid, aspartic acid, sarcosine, trimethylamine, lysine, malonic acid, methanol, carnitine, glycine, glycerol, guanidinoacetic acid, betaine, threonine, malic acid, glucose, galactose, uracil, fumaric acid, tyrosine, phenylalanine, xanthine, and formic acid and eight lipoproteins including total chol, HDLchol, LDL chol, VLDLchol, total chol/HDLchol, total chol/HDL-Apo A, LDLchol/HDLchol and VLDLchol/HDLchol. The correlation analysis was performed separately for low fitness (blue) and high fitness subjects (red).

In order to further investigate a possible relationship between the human fecal metabolome and plasma LP, subjects were sorted according to their plasma concentrations of selected LPs or their ratio, some of which are known to be a risk factor for CVD, and classified either as low-LP (bottom 1/3) or high-LP (top 1/3) subjects (Table S7). Then, the fecal metabolome datasets, both from NMR and GC-MS, were scrutinized to reveal fecal metabolic differences between the low-LP and high-LP subjects. One-way ANOVA identified 16 fecal metabolites being different between the low- and high-LP groups (Table 1). These metabolites included two SCFAs (butyric acid and isovaleric acid), 11 AAs (valine, leucine, isoleucine, alanine, glutamic acid, proline, aspartic acid, lysine, glycine, methionine, and phenylalanine), methanol, lactic acid, and uracil. Almost all the metabolites displayed a higher concentration in the low-LP group, except for the two SCFAs, butyric acid and isovaleric acid. Butyric acid showed an increased concentration in the high-HDLchol group, while isovaleric acid showed an increased concentration in 
the high-VLDLchol group. In addition, the ratios between LP variables, which have been suggested as CVD risk factors [30], such as total chol/HDLchol, total chol/HDL-Apo A, LDLchol/HDLchol, and VLDLchol/HDLchol, were found to be related to the fecal levels of SCFAs, AAs, methanol, and uracil. The level of butyric acid showed increased concentrations in low-LDLchol/HDLchol and VLDLchol/HDLchol groups compared to the corresponding high-LP groups. Amino acids such as leucine, isoleucine, valine, phenylalanine, glycine, lysine, methionine, lactic acid, and uracil had higher concentrations in the low-total-chol/HDL-Apo A group compared to the high-LP-ratio group. In addition, fecal concentrations of valine, alanine, glutamic acid, and methanol were found to be higher in subjects with a low total chol/HDLchol ratio.

Table 1. A list of human fecal metabolites that have significantly different concentrations in older adults according to their blood plasma concentrations of selected lipoproteins. These lipoproteins included total chol, tg, HDLchol, LDLchol, VLDLchol, and the LP ratios of total chol/HDLchol, total chol/HDL-Apo A, LDLchol/HDLchol, and VLDLchol/HDLchol. $\mathrm{MH}=$ mean high LP, ML = mean low LP, total chol = total cholesterol, HDLchol = high-density lipoprotein cholesterol, LDLchol = low-density lipoprotein cholesterol, VLDLchol = very-low-density lipoprotein cholesterol, $t g=$ triglyceride, HDL-Apo A = high-density lipoprotein-Apolipoprotein A, CVD = cardiovascular diseases.

\begin{tabular}{|c|c|c|c|c|}
\hline Metabolite & CVD Risk Factor & $p$-Value & Effect Size (\%) & Fold Change(MH/ML) \\
\hline \multirow{3}{*}{ butyric acid } & HDLchol & 0.038 & 4.02 & 1.28 \\
\hline & LDLchol/HDLchol & 0.033 & 4.20 & 0.78 \\
\hline & VLDLchol/HDLchol & 0.044 & 3.73 & 0.79 \\
\hline \multirow{2}{*}{ isovaleric acid } & HDLchol & 0.001 & 11.08 & 0.80 \\
\hline & VLDLchol & 0.013 & 5.63 & 1.29 \\
\hline \multirow{3}{*}{ leucine } & LDLchol & 0.030 & 4.55 & 0.85 \\
\hline & total chol/HDL-Apo A & 0.016 & 5.28 & 0.84 \\
\hline & $\operatorname{tg}$ & 0.039 & 4.63 & 0.76 \\
\hline \multirow{2}{*}{ valine } & total chol/HDLchol & 0.003 & 9.20 & 0.68 \\
\hline & total chol/HDL-Apo A & 0.022 & 4.80 & 0.84 \\
\hline alanine & total chol/HDLchol & 0.002 & 9.90 & 0.58 \\
\hline \multirow{3}{*}{ glutamic acid } & total chol/HDLchol & 0.020 & 5.02 & 0.67 \\
\hline & LDLchol & 0.011 & 6.17 & 0.67 \\
\hline & LDLchol/HDLchol & 0.025 & 4.68 & 0.68 \\
\hline \multirow{2}{*}{ phenylalanine } & LDLchol & 0.019 & 5.30 & 0.84 \\
\hline & total chol/HDL-Apo A & 0.011 & 5.90 & 0.88 \\
\hline proline & $\operatorname{tg}$ & 0.041 & 4.54 & 0.69 \\
\hline aspartic acid & $\operatorname{tg}$ & 0.004 & 8.67 & 0.48 \\
\hline \multirow{2}{*}{ methanol } & total chol/HDLchol & 0.017 & 5.27 & 0.73 \\
\hline & LDLchol & 0.020 & 5.22 & 0.66 \\
\hline glycine & total chol/HDL-Apo A & 0.020 & 4.96 & 0.85 \\
\hline lysine & total chol/HDL-Apo A & 0.040 & 3.87 & 0.85 \\
\hline \multirow{2}{*}{ methionine } & total chol/HDL-Apo A & 0.031 & 4.27 & 0.78 \\
\hline & LDLchol/HDLchol & 0.038 & 4.01 & 0.80 \\
\hline lactic acid & total chol/HDL-Apo A & 0.045 & 3.71 & 0.74 \\
\hline isoleucine & total chol/HDL-Apo A & 0.023 & 4.74 & 0.84 \\
\hline uracil & total chol/HDL-Apo A & 0.013 & 5.67 & 0.83 \\
\hline
\end{tabular}




\section{Discussion}

One of the key steps to improve the quality of life and well-being of older adults is to better understand factors influencing their physical fitness. In the CALM study, almost half of the participants were either overweight or obese with high total blood cholesterol ( $>5.7 \mathrm{mmol} / \mathrm{L}$ ) levels, which is considered a CVD risk factor. High BMI and total chol in older adults can be explained by low basal metabolism, dietary habits, and lifestyle. On the other hand, only a few of the participants were on the borderline or within the actual risk zone in terms of plasma levels of total $t g$, VLDLchol, LDLchol, HDLchol, and total chol/HDLchol. More than $90 \%$ of the participants were found to be in the normal range in terms of concentrations of the above LP. The roles of gut health and alterations in the fecal metabolome in chronic diseases have been characterized in a few studies, while their relations to physical fitness are still poorly understood. A previous study showed that older adults differing in their physical fitness also have differences in their gut microbiome and glucose metabolism [14].

The current study shows how the fecal metabolome differs between the two fitness groups (LF and HF). The differences were weak but significant and covered a wide range of metabolites, such as SCFAs and AAs, which are important molecules for the maintenance of a healthy gut microbiome. AAs and SCFAs are functional metabolites regulated by the gut microbiome, and SCFAs are generated by bacterial fermentation that is specific to dietary fibers and resistant starch in the colon [31]. They are also found to be the metabolites that differ the most between LF and HF groups. An increasing number of studies have provided evidence that SCFAs also exert important physiological effects on human health $[32,33]$. Ortiz-Alvarez et al. reported that fitness is playing a key role in the secretion of SCFAs, with increased fecal SCFA concentrations found in a long-term exercise intervention group [34]. Barton et al. found that acetic acid, propionic acid, and butyric acid concentrations are increased in the gut of trained subjects (male rugby players) [35]. However, in this study, we can only confirm that the fecal levels of propionic acid and lactic acid were slightly increased in LF subjects compared to HF subjects. This discrepancy may be because the study by Barton et al. was performed as a long-term fitness intervention, while the present study only included baseline fitness levels of free-living community-dwelling older adults who have not (yet) been under specific physical training. Moreover, the older subjects in this study showed significantly lower concentrations of SCFAs than younger, 18-years-old, subjects did, as previously reported [26]. However, the increased concentrations of SCFAs in LF subjects may also be explained by the altered regulation of glucose transport, fatty acid oxidation, and steroid biosynthesis in LF subjects compared to HF subjects [36,37], and this increase has also been related to different rates of glucose homeostasis and lipid metabolism between HF and LF subjects [38].

Fecal AAs were also found at increased concentrations in LF subjects. AAs are the building blocks of proteins and enzymes, and they are partially digested and absorbed in the intestine. It has been shown that LF subjects were eating more protein than the HF subjects [14]. Although this difference in protein consumption does not influence the fecal AA levels, it weakly affects the isovaleric acid and propionic acid levels [26]. In feces, AAs derive from the endogenous metabolism of the gut microbiome [39], and the effect of fitness on changing fecal AA levels is elusive [40]. Deda et al. [39] reported similar results regarding AA changes in feces, with lower levels found after high-exercise training. These differences in AA levels have been suggested to be linked with diet composition, which, in turn, generates the growth of gut microbes such as Firmicutes, which are crucial for AA and carbohydrate metabolism [41]. In addition, physical fitness has also been related to a higher fecal alpha bacteria diversity, further influencing the AA and SCFA levels [34]. Similar to our findings, Kujala et al. showed a lower level of isoleucine in the serum of the higher-fitness subjects compared to lower-fitness subjects. Sakaguchi et al. found that the concentrations of AAs, including leucine, isoleucine, alanine, methionine, lysine, and glutamic acid, in human plasma increase after $14 \mathrm{~h}$ of heavy exercise [42]. 
Ethenyl acetate and oxalic acid that are increased in the feces of HF males compared to LF males are potentially harmful to human health. For example, ethenyl acetate is considered a possible carcinogenic compound [43], and oxalic acid is considered to be a natural antioxidant in some systems [44], while increased levels of AAs in LF subjects compared to HF subjects might be related to the malabsorption in older adults [45].

High BMI is considered a risk factor for T2D, and lowering BMI is important for regulating T2D [46]. In this study, we found 14 fecal metabolites significantly different between low-BMI (<23.5) and high-BMI (>27) subjects. Nogacka et al. found that the number of Bacteroidetes was significantly downregulated in overweight or obese subjects, which may be related to the BMI effects observed in this study [47]. A study by Liang et al. demonstrated that the human fecal metabolome composition can be associated with diabetes, with fecal homocysteine and butanone levels being significantly increased in diabetic patients compared with controls, whereas the levels of L-malic acid and dibutyl decanedioate were decreased in diabetic patients [48]. In agreement with our results, Liang et al. concluded that AA levels in feces are greater in subjects who are at high risk of diabetes compared to lower-risk subjects. In addition, Zhou et al. showed that the fecal level of phenylalanine is higher in feces of T2D patients compared to patients treated against T2D [49]. The elevated levels of certain AAs in older adults with high BMI are hypothesized to be due to impaired metabolism and specific gut microbiota promoted in overweight or obese subjects. The high-BMI subjects are expected to have lower gut microbiota diversity, which may generate specific effects on gut metabolites. Combined, these findings provide evidence that the fecal metabolome can give an indication of impaired metabolism due to obesity, which are primarily pronounced as increased levels of fecal AAs such as valine, alanine, phenylalanine, and tyrosine.

Human blood plasma lipoproteins have long been studied as a 'risk factor' for CVD and liver diseases [50]. The analysis of the CALM cohort showed that high BMI, thus lowfitness, subjects have a decreased level of plasma HDLchol, increased level of LDLchol, and intermediate-density lipoprotein cholesterol (IDLchol) (Trimigno et al., in press). Similar to the findings of Trimigno et al., Pasiakos et al. reported that lower-BMI subjects have higher HDLchol levels at high-protein diets [51], which indicates that high-BMI subjects potentially have a higher risk of CVD as HDLchol is recognized as good cholesterol [52]. The higher risk of CVD for overweight subjects has also been related to impaired HDLchol metabolism [53]. Schmedes et al. concluded that weight loss decreases both the levels of LP in plasma and SCFA in fecal samples [54].

Therefore, the correlations found between fecal metabolites and certain LP levels indicate that research in this direction may provide new insights into a link between human metabolism/health and the gut microbiome. In this paper, a comparison of the fecal metabolite concentrations of two groups (low LP and high LP) was performed. The results indicated that a normal (according to the Danish health care system) concentration range of plasma HDLchol level is associated with an increased fecal butyric acid level compared to those older adults whose plasma HDLchol level was lowered $(<1.1 \mathrm{mmol} / \mathrm{L})$. Subjects with lower HDLchol levels instead showed higher fecal levels of isovaleric acid, suggesting that an increase of this SCFA in the fecal matter may be a sign of a higher risk of CVD. Similarly, Zheng et al. found that the blood lipid and LP levels were negatively correlated with the blood metabolome, SCFAs including acetic acid, butyric acid, and propionic acid in feces were related to blood cholesterol levels in overweight women [3]. Diet may be a key factor explaining the links between LP levels and fecal SCFA as dietary fat and carbohydrate intake have a significant effect on fecal SCFA levels in humans [26]. These findings on LP and the fecal metabolome can potentially provide useful information for the design of a diet that could reduce the risk of CVD in hyperlipidemia patients. In addition, we have documented that LDLchol, $t g$, and cholesterol ratios, including (total chol/HDLchol) and total chol/HDL-Apo A, are related (more sensitive) to the fecal levels of AAs in older adults. This is an interesting finding as AAs are also important building blocks of proteins and, thus, may be key for determining their functions. Our study also showed 
that increased fecal levels of AAs, including leucine, isoleucine, valine, phenylalanine, glycine, lysine, and methionine, were related to a normal (healthy) concentration range of good cholesterol (HDLchol), and thus may be a sign of low risk of CVD. The changes in fecal AA levels may have a close relationship with the transport and reduction of lipids in older adults [55], but may also be linked to diet [26]. Especially, dietary intake of fat might be one of the reasons (confounders) explaining the increased fecal isoleucine and valine levels in high BMI subjects. These specific AAs are likely the most sensitive fecal metabolites reflecting BMI or the levels of plasma LP in humans. Understanding the mechanisms, direct or indirect relationships in between the changes in human fecal metabolome with BMI or LP concentrations, is particularly important to adjust the dietary and lifestyle recommendations for older adults to reduce the risk of CVD. Future studies are required to validate correlations found in this study between the human fecal metabolome and physical fitness, BMI, and plasma LP levels, which will open new perspectives for better understanding the role of the human gut microbiome in the health and well-being of older adults.

\section{Materials and Methods}

\subsection{Sample Collection}

All subjects included in this study were apparently healthy older adults, communitydwelling, and self-supportive. Participants were recruited through local newspapers, magazines, radio programs, social media, and presentations at senior centers and public events [56]. Anthropometric, body-composition, and physical performance measurements, average daily physical activity, dietary intake and preferences, microbiome composition, and clinical biomarkers were recorded for each participant, and fecal samples were collected [14]. A total of 163 subjects (83 males and 80 females) who provided a sufficient amount of fecal samples were included in this study out of 205 subjects recruited in the CALM project. All subjects who participated in the CALM project also provided written informed consent. Procedures of the CALM project (Clinical Trials NCT02115698) were approved by the Danish Regional Ethical Committees of the Capital Region (J-nr. H-42013-070) and performed according to the Declaration of Helsinki II [57]. After collection, fecal samples were immediately stored in the fridge at $4{ }^{\circ} \mathrm{C}$ for a maximum of $48 \mathrm{~h}$ and subsequently stored at $-80{ }^{\circ} \mathrm{C}$ until NMR analysis. The metadata, including sex, age, height $(\mathrm{cm})$, body weight $(\mathrm{kg})$, and individual dietary energy and macronutrient intake, were collected for each participant. Blood samples were collected for measuring VLDLchol, LDLchol, HDLchol, total tg, total chol, and their subclasses. More details of the metadata collected in the CALM project have been previously reported $[14,56]$.

\subsection{Chemicals}

Chemicals and reagents used in this study were purchased from Sigma-Aldrich (Søborg, Denmark). The chemicals included deuterium oxide $\left(\mathrm{D}_{2} \mathrm{O}, 99.9\right.$ atom \% D), monobasic potassium phosphate $\left(\mathrm{KH}_{2} \mathrm{PO}_{4}, \geq 99.0 \%\right)$, dibasic potassium phosphate $\left(\mathrm{K}_{2} \mathrm{HPO}_{4}, \geq 98.0 \%\right)$, sodium salt of 3-(trimethylsilyl) propionic-2,2,3,3-d4 acid (TSP, 98 atom \% D, $\geq 98.0 \%$ ), sodium chloride $(\mathrm{NaCl}, \geq 99.0 \%)$, methanol $\left(\mathrm{CH}_{3} \mathrm{OH}, \geq 99.98 \%\right.$, containing $10 \mathrm{ppm}$ of palmitic-acid methyl ester and $10 \mathrm{ppm}$ of sorbitol as internal standards), and sodium azide $\left(\mathrm{NaN}_{3}, \geq 99.5 \%\right)$. The water used throughout the study was purified using a Millipore lab water system (Merck KGaA, Darmstadt, Germany) equipped with a $0.22 \mu \mathrm{m}$ filter membrane.

\subsection{Sample Preparation for Metabolomics Analysis}

\subsubsection{Fecal Sample Preparation for ${ }^{1} \mathrm{H}$ NMR Metabolomics Analysis}

Fecal samples were thawed at $4{ }^{\circ} \mathrm{C}$ and mixed with ultrapure water at a $w / v$ ratio of 1:2 (feces/water) and transferred into filter bags for homogenizing for $1 \mathrm{~min}$ (Lab Seward, BA7021, Seward, AK, USA); the fecal slurry was then stored at $-80{ }^{\circ} \mathrm{C}$ before further analysis. Before the metabolite extraction for NMR analysis, fecal slurry samples were 
thawed at $4{ }^{\circ} \mathrm{C}$, and $600 \mu \mathrm{L}$ of the slurry was transferred into a $1.5 \mathrm{~mL}$ plastic Eppendorf tube and then mixed with $600 \mu \mathrm{L}$ of phosphate buffer, followed by vortexing for $2 \mathrm{~min}$. The mixture was then centrifuged at $6{ }^{\circ} \mathrm{C}$ for $30 \mathrm{~min}$ at $14,000 \times \mathrm{g}$. Finally, $600 \mu \mathrm{L}$ of clear supernatant was transferred into a $5 \mathrm{~mm}$ O.D. SampleJet NMR tube for ${ }^{1} \mathrm{H}$ NMR analysis. At least one control sample was included in the NMR analysis between 10 real samples. Phosphate buffer was employed as the extraction solvent; details of the fecal sample preparation protocol and phosphate buffer preparation have been described in Cui et al. [16].

\subsubsection{Fecal Sample Preparation for GC-MS Metabolomics Analysis}

A volume of $1 \mathrm{~mL}$ of the homogenized fecal flurry (as described earlier) was mixed with $1 \mathrm{~mL}$ of phosphate buffer, and then quickly frozen in liquid nitrogen and freezedried overnight. Twenty milligrams of the freeze-dried fecal powder sample was then re-suspended in $1 \mathrm{~mL}$ of methanol, vortexed, and centrifuged for $30 \mathrm{~min}$ at $12,000 \times g$ at $4{ }^{\circ} \mathrm{C}$. Fifty microliters of the supernatant was then dried using a ScanVac (Labogene, Lynge, Denmark) at $1000 \mathrm{rpm}$ for $3 \mathrm{~h}$ at $40{ }^{\circ} \mathrm{C}$. Immediately after drying, samples were sealed with airtight magnetic lids into $2.0 \mathrm{~mL}$ GC-MS vials and derivatized in two steps using a Dual-Rail MultiPurpose Sampler (MPS) (Gerstel, Mülheim an der Ruhr, Germany): (i) addition of $10 \mu \mathrm{L}$ of MEOX reagent $\left(20 \mathrm{mg} \mathrm{mL}^{-1}\right.$ of methoxiamine hydrochloride in dry pyridine) followed by agitation at $45^{\circ} \mathrm{C}$ for $90 \mathrm{~min}$ by mixing at $750 \mathrm{rpm}$, (ii) addition of $40 \mu \mathrm{L}$ of TMS reagent, trimethylsilyl cyanide (TMSCN) [58], followed by agitation at $45{ }^{\circ} \mathrm{C}$ for $45 \mathrm{~min}$ by mixing at $750 \mathrm{rpm}$. All steps involving sample derivatization and injection were automated using the MPS, which was equipped with a sample agitation unit. Immediately after derivatization, $1 \mu \mathrm{L}$ of the derivatized sample was injected into a cooled injection system (CIS4) (Gerstel, Mülheim an der Ruhr, Germany) port in splitless mode. More details of the targeted and untargeted GC-MS fecal sample preparation protocol and phosphate buffer preparation have been described by Castro-Mejía et al. [14].

\subsection{Data Acquisition}

\subsection{1. ${ }^{1} \mathrm{H}$ NMR Spectroscopy Analysis}

${ }^{1} \mathrm{H}$ NMR spectra were recorded at the University of Copenhagen (Department of Food Science) using a Bruker Advance III $600 \mathrm{MHz}$ NMR spectrometer equipped with a $5 \mathrm{~mm}$ broadband inverse RT (BBI) probe, automated tuning and matching accessory (ATMA) and cooling unit BCU-05, and an automated sample changer (Sample Jet, Bruker Biospin, Rheinstetten, Germany) with sample cooling (278 K) and preheating stations (298 K). Data acquisition and processing were carried out using TOPSPIN 3.5 PL6 (Bruker Biospin, Rheinstetten, Germany). The Icon NMR (Bruker Biospin, Rheinstetten, Germany) interface was used to control the automation of the overall measurement procedure. Automatic tuning and matching, lock, and shimming were performed using TOPSPIN 3.5 PL6. The standard pulse sequence with water suppression (noesygppr1d) was employed for measuring the one-dimensional ${ }^{1} \mathrm{H}$ NMR spectra. A total of 32 scans were acquired after 4 dummy scans, and the generated free induction decays (FIDs) were collected into $128 \mathrm{k}$ data points using a spectral width of $20 \mathrm{ppm}$. Acquisition time, recycle delay, and mixing time were set to $2.72,4.0$, and $0.01 \mathrm{~s}$, respectively. The receiver gain was set to 90.5 . A more detailed description of NMR analysis has been reported in a previous paper [16].

\subsubsection{GC-MS Data Acquisition}

The GC-MS setup was made by combining an Agilent 7890B gas chromatograph (GC) (Agilent Technologies, Santa Clara, CA, USA) with a time-of-flight mass spectrometer, HT Pegasus TOF-MS, (LECO Corporation, Saint Joseph, MO, USA). A Zebron ZB 5\% Phenyl 95\% dimethylpolysiloxane column (30 m with I.D. $250 \mu \mathrm{m}$ and film thickness $0.25 \mu \mathrm{m}$ ) with a $5 \mathrm{~m}$ inactive guard column (Phenomenex, Torrance, CA, USA) was employed for GC separation. A hydrogen generator, Precision Hydrogen Trace 500 (Peak Scientific Instruments Ltd., Inchinnan, UK), was used to supply a carrier gas at a constant column 
flow rate of $1.0 \mathrm{~mL} \mathrm{~min}^{-1}$. The initial temperature of the GC oven was set to $40{ }^{\circ} \mathrm{C}$, and this temperature was held for $2 \mathrm{~min}$, followed by heating at the rate of $10^{\circ} \mathrm{C} \mathrm{min}-1$ to reach $320^{\circ} \mathrm{C}$, and then held for $5 \mathrm{~min}$ at $320^{\circ} \mathrm{C}$. The mass spectra were recorded at the mass-tocharge $(\mathrm{m} / \mathrm{z})$ range of $45-600$, and the scanning frequency was set to 10 scans s$^{-1}$. The MS detector and ion source were switched off during the first $6.3 \mathrm{~min}$ of solvent delay time, and the transfer line and ion source temperature were set to $280^{\circ} \mathrm{C}$ and $250{ }^{\circ} \mathrm{C}$, respectively. The mass spectrometer was tuned according to the manufacturer's recommendation using perfluorotributylamine (PFTBA). MPS and GC-MS were controlled using vendor software Maestro (Gerstel, Mülheim an der Ruhr, Germany) and ChromaTOF (LECO Corporation, Saint Joseph, MO, USA), respectively. A more detailed description of GC-MS analysis has been reported in a previous paper [14].

\subsection{Processing of the Raw ${ }^{1} H$ NMR Spectra and GC-MS Data}

The acquired ${ }^{1} \mathrm{H}$ NMR spectra were processed using SigMa [59]. First, they were normalized to the Electronic Reference To access In vivo Concentrations (ERETIC) signal [60]. Then, reference alignment to the TSP signal was carried out, followed by pre-alignment of 98 spectral regions. 83 signature signals (SS) and 76 signals of unknown spin-system (SUS), were then identified from the fecal NMR spectra, aligned, and quantified using the SigMa software as described in the previous study [16]. In addition, 49 BINs (intervals of complex regions representing more than one metabolite) were identified and quantified by summing all data points. Absolute concentrations (ACs) of 36 metabolites were calculated in SigMa using the areas of the corresponding SS, a corresponding proportion of a given proton in a metabolite, and the area of the ERETIC signal, which corresponds to $10 \mathrm{mM}$ of protons. Details of the quantification and distributions of metabolites' concentrations can be found in the study by Cui et al. [26].

The raw GC-MS data were processed using the Statistical Compare toolbox of the ChromaTOF software (Version 4.50.8.0, LECO Corporation, Saint Joseph, USA). Deconvoluted mass spectra were used for peak identification using LECO-Fiehn and NIST11 libraries. The library search was set to return the top 10 hits with an EI-MS match of $>75 \%$ using the normal-forward search and with a mass threshold of 20 . Deconvoluted peaks were aligned across all samples using the following settings: retention time shift allowance of $<3$ s, EI-MS match of $>95 \%$, mass threshold of $>25$, and present in $>90 \%$ of all pooled control samples. The details of metabolite identification from GC-MS data have been described in a previous paper [61].

\subsection{Statistical Analysis}

Multivariate analyses, including principal component analysis (PCA) [62], analysis of variance-simultaneous component analysis (ASCA) [63], and partial least squares (PLS) regression [64], as well as one-way ANOVA with Benjamini-Hochberg's multiple test correction, and the FDR (False Discovery Rate) rate of 10\% [65], were performed on NMR and GC-MS metabolite data to find possible effects of physical fitness, BMI, and LP on the human fecal metabolome. All significant metabolites have also been evaluated using the Monte Carlo test $(N=2000)$. Metabolite data were checked for normal distribution prior to ANOVA. All statistical analyses were performed on MATLAB (version R2015a, the Mathworks, Inc., Natick, MA, USA), and customized MATLAB scripts were written by the authors.

\section{Conclusions}

This study investigated the correlations between human fecal metabolome and physical fitness, BMI, and blood plasma concentrations of LP in healthy older adults. Overall, weak correlations were found between fitness and the fecal metabolome, with decreased concentrations of SCFAs (propionic acid and lactic acid) and AAs (methionine, leucine, glutamic acid, and threonine) in the high-fitness group. The effect of BMI on the concentrations of fecal metabolites is stronger, with increased concentrations of AAs (valine, alanine, 
and phenylalanine) and uracil in subjects with a high BMI. When the effect of BMI was investigated separately for low- and high-fitness subjects, main alterations in the fecal metabolome were pronounced on SCFAs and AAs, indicating a different function of the gut microbiome. The weak effects of LP levels and cholesterol ratios on the fecal metabolome were reflected on the levels of SCFAs (butyric acid and isovaleric acid) and AAs (valine, glutamic acid, phenylalanine, etc.). High LDLchol levels were related to decreased levels of valine, glutamic acid, phenylalanine, and lactic acid. We hypothesize that the diet composition, especially protein and fat intakes, is potentially a key factor determining the links between plasma LP levels and fecal SCFA levels, but more mechanistic-oriented studies are needed for validation. Thus, the results obtained in this study help to better understand how physical fitness and health biomarkers such as blood plasma LP are associated with the composition of the human fecal metabolome and, in turn, for linking these with gut microbiota function and human well-being.

Supplementary Materials: The following are available online at https:/ / www.mdpi.com/article/10.339 0/metabo11110717/s1. Figure S1: Distribution of Pearson's correlation coefficients ( $r$-values) calculated between $36 \mathrm{fecal}$ metabolites including valeric acid, isovaleric acid, leucine, isoleucine, valine, propionic acid, ethanol, lactic acid, alanine, butyric acid, acetic acid, methionine, acetone, glutamic acid, pyruvic acid, aspartic acid, sarcosine, trimethylamine, lysine, malonic acid, methanol, carnitine, glycine, glycerol, guanidinoacetic acid, betaine, threonine, malic acid, glucose, galactose, uracil, fumaric acid, tyrosine, phenylalanine, xanthine, and formic acid and eight lipoproteins including total chol, HDLchol, LDLchol, VLDLchol, total chol/HDLchol, total chol/HDL-Apo A, LDLchol/HDLchol and VLDLchol/HDLchol. The correlation analysis was performed separately for (A) males, (B) females, (C) low BMI and (D) high BMI groups. (BMI = body mass index), Figure S2: Heat-Maps made of Pearson's correlation coefficients ( $r$-values) calculated between 36 fecal metabolites including valeric acid, isovaleric acid, leucine, isoleucine, valine, propionic acid, ethanol, lactic acid, alanine, butyric acid, acetic acid, methionine, acetone, glutamic acid, pyruvic acid, aspartic acid, sarcosine, trimethylamine, lysine, malonic acid, methanol, carnitine, glycine, glycerol, guanidinoacetic acid, betaine, threonine, malic acid, glucose, galactose, uracil, fumaric acid, tyrosine, phenylalanine, xanthine, and formic acid and eight lipoproteins including total chol, HDLchol, LDLchol, VLDLchol, total chol/HDLchol, total chol/HDL-Apo A, LDLchol/HDLchol and VLDLchol/HDLchol. The correlation analysis was performed separately for (A) males, (B) females, (C) low BMI and (D) high BMI groups. (BMI = body mass index), Table S1: A list of fecal metabolites identified from the GC-MS data. Metabolites were identified by spectral matching using LECO-Fiehn and NIST11 libraries that are extracted from the NIST11 metabolite database. The table includes the metabolite names, metabolite class (class $1=$ fatty acids, class $2=$ amino acids, class $3=$ organic acid, class $4=$ sugars, class $5=$ sugar alcohols, class $6=$ other metabolites), occurrence (percentage frequency of detection of a metabolite across samples), calculated (in this study) and reported (in NIST11 metabolite library) retention indexes (RI), the difference between the reported RI and the calculated RI $(\Delta R I)$, identification level (Identified according to the Metabolomics Standards Initiative using electron ionization-mass spectrometry and retention index-based matching), EI-MS spectral match (R.Match), mean intensity(\%) and the status of discovery of the metabolites in human fecal, Table S2: The results from the ANOVA analysis performed on NMR (A) and GC-MS (B) based metabolite data to investigate the differences between low fitness and high fitness groups including all subjects. (HF = high fitness, LF = low fitness), Table S3: The results from the ANOVA analysis performed on the sex stratified NMR and GC-MS based metabolite data to investigate the differences between low fitness (LF) and high fitness (HF) groups, Table S4: The results from the ANOVA analysis performed on NMR based metabolite data to investigate the differences between low BMI and high BMI groups including all subjects. $\mathrm{MH}=$ mean high BMI, ML = mean low BMI, Table S5: The results from the ANOVA analysis performed on the sex stratified NMR based metabolite data to investigate the differences between low BMI and high BMI groups, Table S6: The Pearson's correlation coefficients and $p$-values calculated between the 36 fecal metabolites and the eight lipoproteins including total chol, HDLchol, LDLchol, VLDLchol, total chol/HDLchol, total chol/HDL-Apo A, LDLchol/HDLchol and VLDLchol/HDLchol, including all subjects. *Significant correlations, Table S7: The thresholds and numbers of subjects representing top $1 / 3$ and bottom $1 / 3$ according to the selected eight lipoproteins including total chol, HDLchol, LDLchol, VLDLchol, total chol/HDLchol, total chol/HDL-Apo A, LDLchol/HDLchol and VLDLchol/HDLchol. 
Author Contributions: Conceptualization, S.B.E. and B.K.; methodology, M.C. and B.K.; software, B.K.; validation, B.K. and A.T.; formal analysis, M.C. and A.T.; investigation, M.C. and A.T.; data curation, M.C., B.K., J.B., R.L.B., S.R., J.L.C.-M., D.S.N. and L.H.; writing-original draft preparation, M.C. and B.K.; writing-review and editing, M.C., A.T., B.K. and S.B.E.; supervision, B.K. and S.B.E.; project administration, S.B.E.; funding acquisition, S.B.E., D.S.N. and L.H. All authors have read and agreed to the published version of the manuscript.

Funding: This research was funded by the China Scholarship Council (CSC) (CSC201706170084) and the Danish Strategic Research Council/Innovation Foundation Denmark (COUNTERSTRIKE, grant number 4105-00015B).

Institutional Review Board Statement: The study was conducted according to the guidelines of the Declaration of Helsinki II. It has been approved by the Danish Regional Ethical Committees of the Capital Region, which have approved the protocol (J-nr. H-4-2013-070).

Informed Consent Statement: All subjects gave their written informed consent to participate in accordance with the Declaration of Helsinki II.

Data Availability Statement: The data presented in this paper is available on request from the corresponding authors.

Acknowledgments: The present study is part of the Ph.D. work of Mengni Cui and is financially supported by the China Scholarship Council (CSC) and the University of Copenhagen. The authors also wish to thank the financial support through the CALM project (Clinical Trials NCT02115698), which were approved by the Danish Regional Committees of the Capital Region (H-4-2013-070) and support from the Danish Strategic Research Council/Innovation Foundation Denmark. The authors also wish to thank all the involved participants.

Conflicts of Interest: The authors declare no conflict of interest.

\section{References}

1. Karu, N.; Deng, L.; Slae, M.; Guo, A.C.; Sajed, T.; Huynh, H.; Wine, E.; Wishart, D.S. A review on human fecal metabolomics: Methods, applications and the human fecal metabolome database. Anal. Chim. Acta 2018, 1030, 1-24. [CrossRef] [PubMed]

2. Ganobis, C.M.; Al-Abdul-Wahid, M.S.; Renwick, S.; Yen, S.; Carriero, C.; Aucoin, M.G.; Allen-Vercoe, E. 1D 1 H NMR as a Tool for Fecal Metabolomics. Curr. Protoc. Chem. Biol. 2020, 12, e83. [CrossRef] [PubMed]

3. Zheng, H.; Lorenzen, J.K.; Astrup, A.; Larsen, L.H.; Yde, C.C.; Clausen, M.R.; Bertram, H.C. Metabolic Effects of a 24-Week Energy-Restricted Intervention Combined with Low or High Dairy Intake in Overweight Women: An NMR-Based Metabolomics Investigation. Nutrients 2016, 8, 108. [CrossRef] [PubMed]

4. Martin, F.-P.J.; Sprenger, N.; Montoliu, I.; Rezzi, S.; Kochhar, S.; Nicholson, J. Dietary Modulation of Gut Functional Ecology Studied by Fecal Metabonomics. J. Proteome Res. 2010, 9, 5284-5295. [CrossRef]

5. Vernocchi, P.; Del Chierico, F.; Putignani, L. Gut Microbiota Profiling: Metabolomics Based Approach to Unravel Compounds Affecting Human Health. Front. Microbiol. 2016, 7, 1144. [CrossRef]

6. Sokol, H.; Seksik, P.; Rigottier-Gois, L.; Lay, C.; Lepage, P.; Podglajen, I.; Marteau, P.; Doré, J. Specificities of the fecal microbiota in inflammatory bowel disease. Inflamm. Bowel Dis. 2006, 12, 106-111. [CrossRef]

7. Palomino-Schätzlein, M.; Mayneris-Perxachs, J.; Caballano-Infantes, E.; Rodríguez, M.A.; Palomo-Buitrago, M.-E.; Xiao, X.; Mares, R.; Ricart, W.; Simó, R.; Herance, J.R.; et al. Combining metabolic profiling of plasma and faeces as a fingerprint of insulin resistance in obesity. Clin. Nutr. 2020, 39, 2292-2300. [CrossRef]

8. Zhu, T.; Goodarzi, M.O. Metabolites Linking the Gut Microbiome with Risk for Type 2 Diabetes. Curr. Nutr. Rep. 2020, 9, 83-93. [CrossRef]

9. Yoshimoto, S.; Loo, T.M.; Atarashi, K.; Kanda, H.; Sato, S.; Oyadomari, S.; Iwakura, Y.; Oshima, K.; Morita, H.; Hattori, M.; et al. Obesity-induced gut microbial metabolite promotes liver cancer through senescence secretome. Nature 2013, 499, 97-101. [CrossRef]

10. Albillos, A.; de Gottardi, A.; Rescigno, M. The gut-liver axis in liver disease: Pathophysiological basis for therapy. J. Hepatol. 2020, 72, 558-577. [CrossRef]

11. Jenkins, D.J.; Kendall, C.W.; Ransom, T.P. Dietary fiber, the evolution of the human diet and coronary heart disease. Nutr. Res. 1998, 18, 633-652. [CrossRef]

12. Derraik, J.G.B.; Rademaker, M.; Cutfield, W.S.; Pinto, T.E.; Tregurtha, S.; Faherty, A.; Peart, J.M.; Drury, P.L.; Hofman, P.L. Effects of Age, Gender, BMI, and Anatomical Site on Skin Thickness in Children and Adults with Diabetes. PLoS ONE 2014, 9, e86637. [CrossRef]

13. Hu, F.B.; Manson, J.E.; Stampfer, M.J.; Colditz, G.; Liu, S.; Solomon, C.G.; Willett, W.C. Diet, Lifestyle, and the Risk of Type 2 Diabetes Mellitus in Women. N. Eng. J. Med. 2001, 345, 790-797. [CrossRef] 
14. Castro-Mejía, J.L.; Khakimov, B.; Krych, Ł.; Bülow, J.; Bechshøft, R.L.; Højfeldt, G.; Mertz, K.H.; Garne, E.S.; Schacht, S.R.; Ahmad, H.F.; et al. Physical fitness in community-dwelling older adults is linked to dietary intake, gut microbiota, and metabolomic signatures. Aging Cell 2020, 19, e13105. [CrossRef]

15. Moludi, J.; Maleki, V.; Jafari-Vayghan, H.; Vaghef-Mehrabany, E.; Alizadeh, M. Metabolic endotoxemia and cardiovascular disease: A systematic review about potential roles of prebiotics and probiotics. Clin. Exp. Pharmacol. Physiol. 2020, 47, 927-939. [CrossRef] [PubMed]

16. Cui, M.; Trimigno, A.; Aru, V.; Khakimov, B.; Engelsen, S.B. Human Faecal 1H NMR Metabolomics: Evaluation of Solvent and Sample Processing on Coverage and Reproducibility of Signature Metabolites. Anal. Chem. 2020, 92, 9546-9555. [CrossRef] [PubMed]

17. Khakimov, B.; Christiansen, L.D.; Heins, A.; Sørensen, K.M.; Schöller, C.E.G.; Clausen, A.; Skov, T.; Gernaey, K.V.; Engelsen, S.B. Untargeted GC-MS Metabolomics Reveals Changes in the Metabolite Dynamics of Industrial Scale Batch Fermentations of Streptoccoccus thermophilus Broth. Biotechnol. J. 2017, 12, 9. [CrossRef] [PubMed]

18. Aru, V.; Lam, C.; Khakimov, B.; Hoefsloot, H.C.; Zwanenburg, G.; Lind, M.V.; Schäfer, H.; van Duynhoven, J.; Jacobs, D.M.; Smilde, A.K.; et al. Quantification of lipoprotein profiles by nuclear magnetic resonance spectroscopy and multivariate data analysis. TrAC Trends Anal. Chem. 2017, 94, 210-219. [CrossRef]

19. Khakimov, B.; Huub, C.J.H.; Mobaraki, N.; Violetta, A.; Kristensen, M.; Mads, V.L.; Holm, L.; Josué, L.C.; Dennis, S.N.; Doris, M.J.; et al. Human blood lipoprotein predictions from 1H NMR spectra: Protocol, model performances and cage of covariance. Biorxiv 2021. [CrossRef]

20. Mertz, K.H.; Reitelseder, S.; Bechshoeft, R.; Bulow, J.; Højfeldt, G.; Jensen, M.; Schacht, S.R.; Lind, M.V.; A Rasmussen, M.; Mikkelsen, U.R.; et al. The effect of daily protein supplementation, with or without resistance training for 1 year, on muscle size, strength, and function in healthy older adults: A randomized controlled trial. Am. J. Clin. Nutr. 2021, 113, 790-800. [CrossRef] [PubMed]

21. DHS, The Danish Healthcare System. 2003: The Joint Public Health Portal, Sundhed.Dk. Available online: https://www.sundhed. dk (accessed on 15 December 2020).

22. Mooradian, A.D.; Haas, M.J.; Wehmeier, K.R.; Wong, N.C. Obesity-related Changes in High-density Lipoprotein Metabolism. Obesity 2008, 16, 1152-1160. [CrossRef] [PubMed]

23. Rashid, S.; Genest, J. Effect of Obesity on High-density Lipoprotein Metabolism. Obesity 2007, 15, 2875-2888. [CrossRef] [PubMed]

24. Van Gaal, L.F.; Vertommen, J.; De Leeuw, I.H. The in vitro oxidizability of lipoprotein particles in obese and non-obese subjects. Atherosclerosis 1998, 137, S39-S44. [CrossRef]

25. de la Cuesta-Zuluaga, J.; Mueller, N.T.; Álvarez-Quintero, R.; Velásquez-Mejía, E.P.; Sierra, J.A.; Corrales-Agudelo, V.; Carmona, J.A.; Abad, J.M.; Escobar, J.S. Higher Fecal Short-Chain Fatty Acid Levels Are Associated with Gut Microbiome Dysbiosis, Obesity, Hypertension and Cardiometabolic Disease Risk Factors. Nutrients 2019, 11, 51. [CrossRef]

26. Cui, M.; Trimigno, A.; Aru, V.; Rasmussen, M.A.; Khakimov, B.; Engelsen, S.B. Influence of Age, Sex, and Diet on the Human Fecal Metabolome Investigated by 1H NMR Spectroscopy. J. Proteome Res. 2021, 20, 3642-3653. [CrossRef]

27. Sumner, L.W.; Amberg, A.; Barrett, D.; Beale, M.H.; Beger, R.; Daykin, C.A.; Fan, T.W.-M.; Fiehn, O.; Goodacre, R.; Griffin, J.L.; et al. Proposed minimum reporting standards for chemical analysis Chemical Analysis Working Group (CAWG) Metabolomics Standards Initiative (MSI). Metabolomics 2007, 3, 211-221. [CrossRef]

28. Nuttall, F.Q. Body Mass Index: Obesity, BMI, and Health: A Critical Review. Nutr. Today 2015, 50, 117-128. [CrossRef]

29. Haug, A.; Høstmark, A.T.; Spydevold, Ø/. Plasma lipoprotein distribution, faecal cholesterol excretion, and activities of lipoprotein lipase, hepatic lipase and lecithin:cholesterol acyltransferase in rats fed diets rich in sucrose or sunflower oil. Acta Physiol. Scand. 1985, 125, 609-617. [CrossRef]

30. Wilson, P.; D’Agostino, R.; Levy, D.; Belanger, A.M.; Silbershatz, H.; Kannel, W.B. Prediction of coronary heart disease using risk factor categories. Circulation 1998, 97, 1837-1847. [CrossRef]

31. Pascale, A.; Marchesi, N.; Marelli, C.; Coppola, A.; Luzi, L.; Govoni, S.; Giustina, A.; Gazzaruso, C. Microbiota and metabolic diseases. Endocrinilogy 2018, 61, 357-371. [CrossRef]

32. Fung, T.C.; Olson, C.A.; Hsiao, E.Y. Interactions between the microbiota, immune and nervous systems in health and disease. Nat. Neurosci. 2017, 20, 145-155. [CrossRef]

33. Dalile, B.; Van Oudenhove, L.; Vervliet, B.; Verbeke, K. The role of short-chain fatty acids in microbiota-gut-brain communication. Nat. Rev. Gastroenterol. Hepatol. 2019, 16, 461-478. [CrossRef]

34. Ortiz-Alvarez, L.; Xu, H.; Martinez-Tellez, B. Influence of Exercise on the Human Gut Microbiota of Healthy Adults: A Systematic Review. Clin. Transl. Gastroenterol. 2020, 11, e00126. [CrossRef]

35. Barton, W.; Penney, N.C.; Cronin, O.; Garcia-Perez, I.; Molloy, M.G.; Holmes, E.; Shanahan, F.; Cotter, P.D.; O'Sullivan, O. The microbiome of professional athletes differs from that of more sedentary subjects in composition and particularly at the functional metabolic level. Gut Microbiota 2018, 67, 625-633. [CrossRef] [PubMed]

36. Al-Khelaifi, F.; Diboun, I.; Donati, F.; Botrè, F.; Alsayrafi, M.; Georgakopoulos, C.; Suhre, K.; Yousri, N.A.; Elrayess, M.A. A pilot study comparing the metabolic profiles of elite-level athletes from different sporting disciplines. Sports Med.-Open 2018, 4, 1-15. [CrossRef]

37. Kirwan, G.M.; Coffey, V.G.; Niere, J.O.; Hawley, J.; Adams, M.J. Spectroscopic correlation analysis of NMR-based metabonomics in exercise science. Anal. Chim. Acta 2009, 652, 173-179. [CrossRef] [PubMed] 
38. Morrison, D.J.; Preston, T. Formation of short chain fatty acids by the gut microbiota and their impact on human metabolism. Gut Microbes 2016, 7, 189-200. [CrossRef] [PubMed]

39. Deda, O.; Gika, H.; Panagoulis, T.; Taitzoglou, I.; Raikos, N.; Theodoridis, G. Impact of exercise on fecal and cecal metabolome over aging: A longitudinal study in rats. Bioanalytical 2017, 9, 21-36. [CrossRef] [PubMed]

40. Lin, R.; Liu, W.; Piao, M.; Zhu, H. A review of the relationship between the gut microbiota and amino acid metabolism. Amino Acids 2017, 49, 2083-2090. [CrossRef] [PubMed]

41. Tanes, C.; Bittinger, K.; Gao, Y.; Friedman, E.S.; Nessel, L.; Paladhi, U.R.; Chau, L.; Panfen, E.; Fischbach, M.A.; Braun, J.; et al. Role of dietary fiber in the recovery of the human gut microbiome and its metabolome. Cell Host Microbe 2021, 29, 394-407. [CrossRef]

42. Sakaguchi, C.A.; Nieman, D.C.; Signini, É.; Abreu, R.M.; Catai, A.M. Metabolomics-Based Studies Assessing Exercise-Induced Alterations of the Human Metabolome: A Systematic Review. Metabolism 2019, 9, 164. [CrossRef]

43. Bogdanffy, M.S.; Sarangapani, R.; Plowchalk, D.R.; Jarabek, A.; E Andersen, M. A biologically based risk assessment for vinyl acetate-induced cancer and noncancer inhalation toxicity. Toxicol. Sci. 1999, 51, 19-35. [CrossRef] [PubMed]

44. Kayashima, T.; Katayama, T. Oxalic acid is available as a natural antioxidant in some systems. Biochim. Et Biophys. Acta-Gen. Sub. 2002, 1573, 1-3. [CrossRef]

45. Holt, P.R. Intestinal Malabsorption in the Elderly. Dig. Dis. 2007, 25, 144-150. [CrossRef] [PubMed]

46. Kok, P.; Seidell, J.C.; E Meinders, A. [The value and limitations of the body mass index (BMI) in the assessment of the health risks of overweight and obesity]. Ned. Tijdschr. voor Geneeskd. 2004, 148, 2379-2382.

47. Salazar, N.; González, S.; Nogacka, A.M.; Rios-Covián, D.; Arboleya, S.; Gueimonde, M.; Reyes-Gavilán, C.G.D.L. Microbiome: Effects of Ageing and Diet. Microbiota Curr. Res. Emerg. Trends 2019, 36, 33-61.

48. Liang, S.; Hou, Z.; Li, X.; Wang, J.; Cai, L.; Zhang, R.; Li, J. The fecal metabolome is associated with gestational diabetes mellitus. RSC Adv. 2019, 9, 29973-29979. [CrossRef]

49. Zhou, Y.; Men, L.; Pi, Z.; Wei, M.; Song, F.; Zhao, C.; Liu, Z. Fecal Metabolomics of Type 2 Diabetic Rats and Treatment with Gardenia jasminoides Ellis Based on Mass Spectrometry Technique. J. Agric. Food Chem. 2018, 66, 1591-1599. [CrossRef]

50. Jiang, J.; Nilsson-Ehle, P.; Xu, N. Influence of liver cancer on lipid and lipoprotein metabolism. Lipids Health Dis. $2006,5,4$. [CrossRef]

51. Pasiakos, S.M.; Lieberman, H.R.; Fulgoni, I.V.L. Higher-Protein Diets Are Associated with Higher HDL Cholesterol and Lower BMI and Waist Circumference in US Adults. J. Nutr. 2015, 145, 605-614. [CrossRef]

52. Xu, S.; Liu, Z.; Liu, P. HDL cholesterol in cardiovascular diseases: The good, the bad, and the ugly? Int. J. Cardiol. 2013, 168, 3157-3159. [CrossRef]

53. Stadler, J.; Lackner, S.; Mörkl, S.; Trakaki, A.; Scharnagl, H.; Borenich, A.; Wonisch, W.; Mangge, H.; Zelzer, S.; Meier-Allard, N.; et al. Obesity Affects HDL Metabolism, Composition and Subclass Distribution. Biomedical 2021, 9, 242.

54. Schmedes, M.S.; Yde, C.C.; Svensson, U.; Håkansson, J.; Baby, S.; Bertram, H.C. Impact of a 6-week very low-calorie diet and weight reduction on the serum and fecal metabolome of overweight subjects. Eur. Food Res. Technol. 2015, 240, 583-594. [CrossRef]

55. Lerin, C.; Goldfine, A.B.; Boes, T.; Liu, M.; Kasif, S.; Dreyfuss, J.; De Sousa-Coelho, A.L.; Daher, G.; Manoli, I.; Sysol, J.R.; et al. Defects in muscle branched-chain amino acid oxidation contribute to impaired lipid metabolism. Mol. Metab. 2016, 5, 926-936. [CrossRef]

56. Bechshøft, R.L.; Reitelseder, S.; Højfeldt, G.; Castro-Mejía, J.L.; Khakimov, B.; Bin Ahmad, H.F.; Kjær, M.; Engelsen, S.B.; Johansen, S.M.B.; Rasmussen, M.A.; et al. Counteracting Age-related Loss of Skeletal Muscle Mass: A clinical and ethnological trial on the role of protein supplementation and training load (CALM Intervention Study): Study protocol for a randomized controlled trial. Trials 2016, 17, 1-17. [CrossRef]

57. Thatte, U.; Puri, K.; Suresh, K.; Gogtay, N. Declaration of Helsinki, 2008: Implications for stakeholders in research. J. Postgrad. Med. 2009, 55, 131-134. [CrossRef]

58. Khakimov, B.; Motawia, M.S.; Bak, S.; Engelsen, S.B. The use of trimethylsilyl cyanide derivatization for robust and broadspectrum high-throughput gas chromatography-mass spectrometry based metabolomics. Anal. Bioanal. Chem. 2013, 405, 9193-9205. [CrossRef]

59. Khakimov, B.; Mobaraki, N.; Trimigno, A.; Aru, V.; Engelsen, S.B. Signature Mapping (SigMa): An efficient approach for processing complex human urine 1H NMR metabolomics data. Anal. Chim. Acta 2020, 1108, 142-151. [CrossRef] [PubMed]

60. Michel, N.; Akoka, S. The application of the ERETIC method to 2D-NMR. J. Magn. Reson. 2004, 168, 118-123. [CrossRef] [PubMed]

61. Khakimov, B.; Poulsen, S.K.; Savorani, F.; Acar, E.; Gürdeniz, G.; Larsen, T.M.; Astrup, A.; Dragsted, L.O.; Engelsen, S.B. New Nordic Diet versus Average Danish Diet: A Randomized Controlled Trial Revealed Healthy Long-Term Effects of the New Nordic Diet by GC-MS Blood Plasma Metabolomics. J. Proteome Res. 2016, 15, 1939-1954. [CrossRef] [PubMed]

62. Hotelling, H. Analysis of a complex of statistical variables into principal components. J. Educ. Psychol. 1933, 24, 417-441. [CrossRef]

63. Smilde, A.K.; Jan, V.D.G.; Hoefsloot, H.C.J.; Lamers, R.-J.A.N.; Van Der Greef, J.; E Timmerman, M. ANOVA-simultaneous component analysis (ASCA): A new tool for analyzing designed metabolomics data. Bioinformatics 2005, 21, 3043-3048. [CrossRef] [PubMed]

64. Ståhle, L.; Wold, S. Partial least squares analysis with cross-validation for the two-class problem: A Monte Carlo study. J. Chemom. 1987, 1, 185-196. [CrossRef]

65. Chumbley, J.R.; Friston, K. False discovery rate revisited: FDR and topological inference using Gaussian random fields. NeuroImage 2009, 44, 62-70. [CrossRef] [PubMed] 